\section{Draft Genome Sequence Resource for Blumeriella jaapii, the Cherry Leaf Spot Pathogen}

\author{
Jingyu Peng, ${ }^{1}$ J. Alejandro Rojas, ${ }^{2,3}$ Hyunkyu Sang, ${ }^{4}$ Tyre J. Proffer, ${ }^{1}$ Cory A. Outwater, ${ }^{1}$ Rytas \\ Vilgalys, ${ }^{2}$ and George W. Sundin ${ }^{1, \dagger}$ \\ ${ }^{1}$ Department of Plant, Soil, and Microbial Sciences, Michigan State University, East Lansing, MI, U.S.A. \\ ${ }^{2}$ Department of Biology, Duke University, Durham, NC, U.S.A. \\ ${ }^{3}$ Department of Entomology and Plant Pathology, University of Arkansas, Fayetteville, AR, U.S.A. \\ ${ }^{4}$ Department of Biotechnology, Chonnam National University, Gwangju, South Korea
}

\begin{abstract}
Blumeriella jaapii is the causal agent of cherry leaf spot (CLS), the most important disease of tart cherry in the Midwestern United States. Infection of leaves by B. jaapii leads to premature defoliation, which places trees at heightened risk of winter injury and death. Current management of CLS relies primarily on the application of three important fungicide classes, quinone outside inhibitors, sterol demethylation inhibitors, and succinate dehydrogenase inhibitors. Here, we present the first high-quality genome of $B$. jaapii through a hybrid assembly of PacBio long reads and lllumina short reads. The assembled draft genome of B. jaapii is $47.4 \mathrm{Mb}$ and consists of 95 contigs with a N50 value of $1.5 \mathrm{Mb}$. The genomic information of $B$. jaapii, representing the most complete sequenced genome of the family Dermateaceae (Ascomycota) to date, provides a valuable resource for identifying fungicide resistance mechanisms of this pathogen and expands our knowledge of the phytopathogenic fungi in this family.
\end{abstract}

\section{Genomic Resource Announcement}

Cherry leaf spot (CLS), caused by Blumeriella jaapii (Rehm) Arx, is the most important limiting factor for the production of tart cherries (Prunus cerasus L.) in the Midwestern United States. Without proper management, CLS infection leads to leaf chlorosis, early leaf defoliation, and poor coloration of fruits. The cultivar Montmorency, which represents $95 \%$ of tart cherry production in Michigan, is highly susceptible to CLS, and unmanaged trees can be $100 \%$ defoliated within 2 months after the first observation of leaf spot symptoms (Andersen et al. 2018). Management of CLS in Michigan, the largest producer of tart cherry in the United States, relies heavily on the use of fungicides in the quinone outside inhibitor (Qol), sterol demethylation inhibitor (DMI), and succinate dehydrogenase inhibitor (SDHI) classes. However, resistance to DMI and SDHI fungicides has become increasingly prevalent in B. jaapii populations (Outwater et al. 2019; Proffer et al. 2006). The DMl fungicide fenbuconazole resistance in $B$. jaapii has been correlated to the insertion of a long interspersed nuclear element (LINE)-like retrotransposon in the promoter sequence of the CYP51 target gene that results in overexpression, and overproduction of the DMI target enzyme, $14 \alpha$-demethylase (Ma et al. 2006). The resistance mechanism of B. jaapii to the SDHI fungicide boscalid has been recently characterized to be associated with a transversion mutation in the succinate dehydrogenase $\mathrm{B}(\mathrm{Sdh} B)$ gene that leads to a substitution of a

\footnotetext{
${ }^{\dagger}$ Corresponding author: G. W. Sundin; sundin@msu.edu

*The $\boldsymbol{e}$-Xtra logo stands for "electronic extra" and indicates that one supplementary table and supplementary materials are published online.
}

The author(s) declare no conflict of interest.

e-X tra* $^{*}$

Funding

This project was funded by the Michigan Cherry Committee.

Keyword

Mycology

Accepted for publication 20 April 2020.

(c) 2020 The American Phytopathological Society 
Table 1. Statistics of assembly summary, gene prediction, and functional annotation for Blumeriella jaapii genome

\begin{tabular}{lc} 
Features & Statistics \\
Genome size (bp) & $47,419,685$ \\
GC content (\%) & $43.0 \%$ \\
Contigs & 95 \\
N50 (bp) & $1,484,584$ \\
Coverage & $56 \times$ \\
BUSCO & $98.6 \%$ \\
Protein-encoding genes & 7,898 \\
NCBI-nr & 6,845 \\
Swiss-Prot & 2,579 \\
KEGG & 6,822 \\
KOG & 1,787 \\
TCDB & 355 \\
GO & 4,841 \\
tRNA & 124 \\
rRNA & 32 \\
Transposable elements & \\
\hline a & 11,764 \\
\hline a Benchmarking universal single-copy orthologs analysis with Ascomycota odb9 database (https:// \\
busco.ezlab.org/).
\end{tabular}

histidine residue for an arginine residue at amino acid 260 (Outwater et al. 2019). Due to the lack of genomic resources for $B$. jaapii, the amplification and identification of fungicide target genomic regions has relied solely on degenerate oligonucleotide primers designed based on the highly conserved genomic regions with known sequences in other fungi (Ma et al. 2006; Outwater et al. 2019). This is technically inconvenient and may not fully resolve the targeting genomic regions.

B. jaapii and its closely related species Marssonina brunnea are members of Dermateaceae, an important family containing multiple important phytopathogens and root-associated endophytes (Bonito et al. 2016). The genetic basis for determining the lifestyle of fungi in Dermateaceae is largely unknown. Currently, only a few genome resources of Dermateaceae are available, including Diplocarpon rosae (Neu et al. 2017), M. brunnea (Zhu et al. 2012), M. coronariae, Pezicula radicicola (Yue et al. 2018), and Pirottaea palmicola (Johnston et al. 2019). The genome sizes of the sequenced Dermateaceae fungi range from 51.9 to $67.6 \mathrm{Mb}$ with GC contents of 42.5 to $45.5 \%$.

The B. jaapii strain 11BO-GW45 used for whole-genome sequencing was previously isolated in 2011 from a 'Montmorency' tart cherry tree on Mahaleb rootstock with CLS symptoms in a commercial orchard in Grand Traverse County in northern Michigan. This strain was validated to be resistant to fenbuconazole. B. jaapii 11BO-GW45 was initially grown on modified malt extract agar (MMEA) medium ( $2 \%$ malt extract, $0.1 \%$ yeast extract, and $2 \%$ agar) and transferred into modified-Melin-Norkans (MMN) broth (per liter: malt extract, $3 \mathrm{~g}$; glucose, $10 \mathrm{~g}$; $\left(\mathrm{NH}_{4}\right)_{2} \mathrm{HPO}_{4}, 0.25 \mathrm{~g} ; \mathrm{KH}_{2} \mathrm{PO}_{4}, 0.5 \mathrm{~g} ; \mathrm{MgSO}_{4} \cdot 7 \mathrm{H}_{2} \mathrm{O}, 0.15 \mathrm{~g} ; \mathrm{CaCl}_{2} \cdot 2 \mathrm{H}_{2} \mathrm{O}, 0.067 \mathrm{~g} ; 1 \mathrm{ml}$ of $1 \%$ $\mathrm{FeCl}_{3} ; \mathrm{NaCl}, 0.025 \mathrm{~g}$; and thiamine $\mathrm{HCl}, 0.1 \mathrm{mg}$ ) and grown for 1 week at room temperature with shaking. We followed Schwessinger's method to isolate high-molecular weight genomic DNA of high quality (Schwessinger and Rathjen 2017). Genomic DNA was sequenced on both PacBio (Pacific Biosciences) and Illumina (Illumina Inc.) platforms to produce long and short reads, respectively. The PacBio RSII library was sequenced using one SMRT cell, achieving $2,639,419,632$ bp of data and nearly $56 \times$ coverage of the genome. For Illumina sequencing, a $2 \times 250$ bp library was generated and sequenced on Illumina MiSeq. PacBio reads were selfcorrected, trimmed, and de novo assembled using Canu (Koren et al. 2017). The draft assembly was further corrected with the PacBio raw reads using Racon (Vaser et al. 2017). Illumina raw reads containing adaptor sequences or in low quality (average Phred score $<30$ ) were filtered via Trimmomatic v0.33 (Bolger et al. 2014). Pilon (Walker et al. 2014) was used to polish the assembled genome with the filtered Illumina reads. 
The resulting genome is $47.4 \mathrm{Mb}$ and contains 95 contigs with an N50 value of $1,484,584 \mathrm{bp}$; the genome is slightly smaller in size than that of its close relative Marssonina brunnea, $51.9 \mathrm{Mb}$ (Zhu et al. 2012). The completeness of genome assembly was assessed via BUSCO v3 (Waterhouse et al. 2017), identifying $98.6 \%$ of the universal single-copy orthologs of Ascomycota. Protein-encoding genes were predicted with GLEAN combining both evidencebased and ab initio approaches (Elsik et al. 2007), and the deduced amino acid sequences were functionally annotated using BLASTP (identity $\geq 40 \%$ and e-value $\leq 1 \times 10^{-5}$ ) against the following databases: NCBI-nr, Swiss-Prot, Kyoto Encyclopedia of Genes and Genomes (KEGG), Eukaryotic Orthologous Groups (KOG), Transporter Classification Database (TCDB), and Gene Ontology (GO), respectively (Table 1). Ribosomal RNA (rRNA) and transfer RNA (tRNA) sequences were identified with rRNAmmer (Lagesen et al. 2007) and tRNAscan-SE (Lowe and Eddy 1997), respectively. Transposable elements were predicted with RepeatMasker version 3.3.0 (http://www.repeatmasker.org/; Tarailo-Graovac and Chen 2009) by aligning the wholegenome sequence against the Repbase database (Jurka et al. 2005). The annotation file of the assembly in GFF3 format is in Supplementary Material. We Sanger-sequenced the succinate dehydrogenase genes, $S d h B, S d h C$, and $S d h D$, with the oligonucleotide primers listed in Supplementary Table S1, and no mismatch was identified (data not shown). We were able to detect the full-length LINE-like retrotransposon (DQ389081.1) immediately upstream of the coding region of the CYP51 14 $\alpha$-demethylase gene, consistent with the observation of fenbuconazole resistance in this strain. The whole genome sequence of $B$. jaapii 11BO-GW45 provides an important resource for identifying the molecular basis of fungicide resistance in this organism. The increased availability of genomic resources in the family Dermateaceae makes it possible to perform comparative genomic studies to understand the plant-fungal interactions in this family and characterize the evolution of shared and lineage-specific virulence factors of phytopathogenic fungi in Helotiales.

Accession numbers. The whole-genome shotgun project is available at GenBank under the accession number VWND00000000, with the associated BioProject PRJNA565027 and BioSample SAMN12730768. The PacBio and Illumina raw data were deposited to NCBI SRA under the accession number SRR10100535 and SRR10100536, respectively. A voucher specimen of B. jaapii 11BO-GW45 has been submitted to the U.S. National Fungus Collections under the accession number BPI 911206.

\section{Acknowledgments}

We thank the Center for Genomic and Computational Biology at Duke University and the Research Technology Support Facility at Michigan State University for sequencing service.

\section{Literature Cited}

Andersen, K. L., Sebolt, A. M., Sundin, G. W., and lezzoni, A. F. 2018. Assessment of the inheritance of resistance and tolerance in cherry (Prunus sp.) to Blumeriella jaapii, the causal agent of cherry leaf spot. Plant Pathol. 67:682-691.

Bolger, A. M., Lohse, M., and Usadel, B. 2014. Trimmomatic: A flexible trimmer for Illumina sequence data. Bioinformatics 30:2114-2120.

Bonito, G., Hameed, K., Ventura, R., Krishnan, J., Schadt, C. W., and Vilgalys, R. 2016. Isolating a functionally relevant guild of fungi from the root microbiome of Populus. Fungal Ecol. 22:35-42.

Elsik, C. G., Mackey, A. J., Reese, J. T., Milshina, N. V., Roos, D. S., and Weinstock, G. M. 2007. Creating a honey bee consensus gene set. Genome Biol. 8:R13.

Johnston, P. R., Quijada, L., Smith, C. A., Baral, H.-O., Hosoya, T., Baschien, C., Pärtel, K., Zhuang, W.-Y., Haelewaters, D., Park, D., Carl, S., López-Giráldez, F., Wang, Z., and Townsend, J. P. 2019. A multigene phylogeny toward a new phylogenetic classification of Leotiomycetes. IMA Fungus 10:1.

Jurka, J., Kapitonov, V. V., Pavlicek, A., Klonowski, P., Kohany, O., and Walichiewicz, J. 2005. Repbase Update, a database of eukaryotic repetitive elements. Cytogenet. Genome Res. 110:462-467.

Koren, S., Walenz, B. P., Berlin, K., Miller, J. R., Bergman, N. H., and Phillippy, A. M. 2017. Canu: Scalable and accurate long-read assembly via adaptive k-mer weighting and repeat separation. Genome Res. 27:722-736.

Lagesen, K., Hallin, P., Rødland, E. A., Stærfeldt, H. H., Rognes, T., and Ussery, D. W. 2007. RNAmmer: Consistent and rapid annotation of ribosomal RNA genes. Nucleic Acids Res. 35:3100-3108.

Lowe, T. M., and Eddy, S. R. 1997. tRNAscan-SE: A program for improved detection of transfer RNA genes in genomic sequence. Nucleic Acids Res. 25:955-964.

Ma, Z., Proffer, T. J., Jacobs, J. L., and Sundin, G. W. 2006. Overexpression of the $14 \alpha-$ demethylase target gene (CYP51) mediates fungicide resistance in Blumeriella jaapii. Appl. Environ. Microbiol. 72:2581-2585.

Neu, E., Featherston, J., Rees, J., and Debener, T. 2017. A draft genome sequence of the rose black spot fungus Diplocarpon rosae reveals a high degree of genome duplication. PLOS One 12:e0185310.

Outwater, C. A., Proffer, T. J., Rothwell, N. L., Peng, J., and Sundin, G. W. 2019. Boscalid resistance in Blumeriella jaapii: Distribution, effect on field efficacy, and molecular characterization. Plant Dis. 103:1112-1118.

Proffer, T. J., Berardi, R., Ma, Z., Nugent, J. E., Ehret, G. R., McManus, P. S., Jones, A. L., and Sundin, G. W. 2006. Occurrence, distribution, and polymerase chain reaction-based detection of resistance to sterol demethylation inhibitor fungicides in populations of Blumeriella jaapii in Michigan. Phytopathol. 96:709-717.

Schwessinger, B., and Rathjen, J. P. 2017. Extraction of high molecular weight DNA from fungal rust spores for long read sequencing. Methods Mol. Biol. 1659: 49-57.

Tarailo-Graovac, M., and Chen, N. 2009. Using RepeatMasker to identify repetitive elements in genomic sequences. Current Protocols in Bioinformatics 25: 4.10.1-4.10.14

Vaser, R., Sović, I., Nagarajan, N., and Šikic, M. 2017. Fast and accurate de novo genome assembly from long uncorrected reads. Genome Res. 27: 737-746. 
Walker, B. J., Abeel, T., Shea, T., Priest, M., Abouelliel, A., Sakthikumar, S., Cuomo, C. A., Zeng, Q., Wortman, J., Young, S. K., and Earl, A. M. 2014. Pilon: An integrated tool for comprehensive microbial variant detection and genome assembly improvement. PLoS One 9:e112963.

Waterhouse, R. M., Seppey, M., Simão, F. A., Manni, M., loannidis, P., Klioutchnikov, G., Kriventseva, E. V., and Zdobnov, E. M. 2017. BUSCO applications from quality assessments to gene prediction and phylogenomics. Mol. Biol. Evol. 35:543-548.
Yue, Q., Li, Y., Chen, L., Zhang, X., Liu, X., An, Z., and Bills, G. F. 2018. Genomicsdriven discovery of a novel self-resistance mechanism in the echinocandinproducing fungus Pezicula radicicola. Environ. Microbiol. 20:3154-3167.

Zhu, S., Cao, Y. Z., Jiang, C., Tan, B. Y., Wang, Z., Feng, S., Zhang, L., Su, X. H., Brejova, B., Vinar, T., Xu, M., Wang, M. X., Zhang, S. G., Huang, M. R., Wu, R., and Zhou, Y. 2012. Sequencing the genome of Marssonina brunnea reveals funguspoplar co-evolution. BMC Genomics 13:382. 